\title{
Devanagari Handwritten Character Recognition using Hybrid Features Extraction and Feed Forward Neural Network Classifier (FFNN)
}

\author{
Saniya Ansari \\ Research Scholar Dept. of ECE, \\ Karpagam University, Coimbatore, India
}

\author{
Udaysingh Sutar, $\mathrm{PhD}$ \\ Research Guide, Dept. of ECE, \\ Karpagam University, Coimbatore, India
}

\begin{abstract}
The process of recognizing scanned documents or machine printed documents using automated or semi-automated tools are resulted into wide range of applications in different real life domains. There are different techniques already introduced by various authors for efficient and accurate recognition of handwritten characters. As designing a method with $100 \%$ accuracy of character recognition is challenging and unachievable task for researchers due to presence of noise, distinct styles of font under real time environment, therefore it is required to design recognition method by considering these characteristics of character recognition. This paper presenting online handwritten recognition framework by using efficient hybrid features codebook and Feed forward neural network (FFNN) to improve the recognition accuracy over Devanagari scripts. Along with the accuracy, another term which plays vital role of deciding the efficiency of recognition method is time required for recognition. Previous techniques giving the more accuracy for recognition, however feature extraction process takes longer time. Therefore such methods failed in real time applications. This paper majorly focusing on different recognition methods previously used and there recognition results, and then presenting our recognition method with its practical results for analysis. The results are varying by considering different image size in MATLAB.
\end{abstract}

\section{Keywords}

Handwritten Recognition, Feature extraction, Devanagari Script, FFNN, SVN, KNN

\section{INTRODUCTION}

The use of electronic documents in modern communication is common and most preferable ways of information sharing which is known as soft copies. These soft copies of documents are having many applications in different fields of real time environment. With use of such soft copies resulted into immediate, secure and easy medium of documents storage and sharing. But still most of communications are used of physical documents like hand written document. Hence the requirement of having automated tool to analyse this handwritten documents accurately is needed. The input handwritten physical documents first required to scan by digital media, then retrieve, analyse, and recognize the characters for further use. The approach used to analyse handwritten documents are falls into the research domain of document image processing. Document image processing is most widely used mechanism in real time applications of different fields like forensic analysis, banking, education, security etc.

The concept of document image analysis is nothing but the extraction of characters from input scanned image of document. Optical character recognition term is used to extract and recognize such image documents. OCR is dealing with scanning handwritten text document by electronic machine. However the existing techniques of OCR required knowing about type of document image script before recognition. And this is default consideration while using OCR. But at real time applications, all end users are not aware about document script type and hence resulted into impractical, undesired approach for character recognition. In short OCR technique is need expert intervention to select the type of script for accurate recognition. In such case if input document is complex means combination of two or more different scripts, then it becomes again more hectic, time consuming tasks of document image analysis as OCR needs to choose particular type of script before proceeding to recognition. To mitigate such issues, recently different automated methods presented for efficient document image analysis and recognition.

In this paper domain of handwritten character recognition for Devanagari script is focused particularly. Methods for offline handwritten recognitions are introduced previously by many researchers. However limited number of study presented for online handwritten recognition. Online handwritten character recognition is dynamic and needs immediate and accurate recognition of input scanned document. Therefore online handwritten character recognition becomes most dominating research domain for researchers in recent years. Under real time applications, such techniques required to work accurately, faster and efficiently to provide fruitful information to end users as end users are heavily depends on such automated tools of handwritten recognition.

The process of recognition characters of online handwritten images is consisting on three main steps such as Image segmentation, extraction of features and finally classification. For each step different methods and algorithms are used. In first phase which consisting of three phases such as image acquisition, image processing and image segmentation. In image acquisition, input scanned handwritten document is retrieved for further processing and read its information. On retrieved image is then pre-processed for image smoothing and noise removal. Here first input image is resized; RGB image converted into grayscale, then noise removed using Gaussian filter technique to segmentation. Next step of first phase is to do image segmentation. Segmentation is basically required to extract only text data and skip all irrelevant information from image. For segmentation morphological operations are used to accurately extract all characters from input handwritten image.

The next phase is to do feature extraction from segmented handwritten image. The accuracy of character recognition is mainly depends on type of features extracted from segmented 
image. More the features extracted, more the accuracy generated. For proposed work different types of existing feature extraction techniques are used and form one new optimized and hybrid feature extraction method. The features like gradient features, projection histograms, zoning, geometric features, structural features etc. frequently used for recognition purpose. Some of the methods of feature extraction takes longer time, but resulting into more accuracy. For real time applications, it is necessary that recognition system should be reliable, faster and accurate. Therefore to overcome the limitations related to time and accuracy, in this work 91 feature vectors are used for each handwritten image with goal of speed operations.

The third and final phase for online handwritten character recognition is classification. There are many classifiers are presented to use for recognition in this domain such as SVM, $\mathrm{KNN}$, ANN, here an efficient and new FFNN classifier is used. The performance metrics used for comparative analysis are false positive rate, false negative rate, f-score, accuracy and recognition time.

Reminder of this paper, section II discussing the related works over handwritten character recognition. Section III is presenting detailed overview of proposed framework of online handwritten character recognition. In section IV the practical analysis and comparative results with discussions are presented. Finally conclusion and future work is predicted in section $\mathrm{V}$.

\section{LITERATURE SURVEY}

In this section different methods used for handwritten character recognition using different classifiers are discussed.

In [1], authors S.P. Kosbatwa and S.K. Pathan, presented how to use classifier ANN (artificial neural network) in the applications those are willing to simplify the code and hence are achieving recognition quality higher. In addition to this, another advantage of using ANN is that extensibility of the system means it allows us to recognize flexible number of character sets rather than fixed. This method is used for problem of pattern classification and solving. Pattern recognition by using method of back propagation is necessary and hence allows optimizing association of input pattern to the output pattern in the neural network.

In [2], author Kauleshwar Prasad author presented method for English character recognition. The English character from input scanned document is recognized by using neural networks. In this paper author presented extensive study over different feature based methods of classifications for recognition of offline English character. In optical character recognition feature extraction is most important phase. The current methods for automatic handwritten recognition performing well either in achieving speed efficiency or achieving accuracy efficiency, but not both. But these methods are replacing existing methods of OCR like for English script. Therefore in [2], author is introduced simple, accurate method for recognizing optical characters by using the neural networks.

In [3], author Sunith Bandaru proposed multi-layered neural network classification method for recognizing the handwritten characters. From the results showing in paper, the simulation of this work is done by using MATLAB GUI. Author designed this GUI in such way that end user can either test or train network on the basis of at time one character. The size of feature vector used for this method is 6 and this satisfying to achieve accurate identification of characters with multi layered neural network.

In [4], author Muhammad Naeem Ayyaz introduced the new handwritten character recognition system using hybrid feature extraction method and support vector machine (SVM). This system is composed of three phases such as preprocessing, extraction of features and training as well as classification using SVM. Author used hybrid feature extraction approach which resulted into more accuracy. These features are able to extract local as well as global variations in handwritten character font styles. The extracted feature vector was a combination of correlation function based features and some statistical/structural features. But the limitation of this method is that it takes more time for recognition.

In [5], author Anita Pal presented that neural network is having vital role for handwritten character recognition. There are many methods presented in literature for English character recognition with goal of achieving more recognition accuracy and less training time, but it is still open problem for researchers using neural network. Hence it is required to design automatic efficient handwritten recognition method for English characters. Therefore author of this paper presented new method based on above said its problem definition. The proposed method in [5] delivers automatic handwritten recognition with better recognition accuracy as well as less training and classification time.

In [6], author proposed method for recognition of Devnagari handwritten numerals using Multi-Layer Perceptron (MLP) neural network classification method. This approach obtained $91.28 \%$ results.

In [7], authors presented the directional chain code features are used with quadratic classifier and resulted into $80.36 \%$ of overall recognition accuracy for handwritten Devnagari characters.

In [8], author present recent method for Devnagari script character recognition using genetic algorithm, this method showing accuracy of $97 \%$ in an average.

\section{PROPOSED METHODOLOGY 3.1 Introduction}

The goal of this paper is to present new robust, efficient and faster method for online Devanagari handwritten character recognition. The proposed framework is composed of three different phases: segmentation, feature extraction and classification. To improve accuracy and reduce the recognition time hybrid feature vector is used which is optimised in terms of time and faster classification method called FFNN. Below section showing the each phase detailed methods used.

In this paper comparative analysis of proposed method against existing SVM and $\mathrm{KNN}$ based recognition systems is presented.

\subsection{Segmentation: Methods Used}

For segmentation input handwritten image is taken as input image, and then it will be resized at size $512 * 512$. Input image is in the form of RGB, which has to be converted it into grayscale.

During pre-processing, Gaussian filtering technique is applied to remove noises. The output of pre-processing is then given to segmentation using binarization method. After binarization, canny edge detection, dilation, erosion is applied. These are 
morphological operators used to remove unwanted pixels before generating final segmentation output. After morphological operations, the output is treated as segmentation results; each character in image is showing in separate bounding box.

\subsection{Feature Extraction: Methods Used}

In this paper novel feature extraction method introduced in which words and characters from handwritten images are considered as unit and further used for recognition. The hybrid feature vector is generated using below listed different types of feature extraction techniques.

- Geometric features.

- Regional Features.

- Gradient Features.

- Distance transforms features.

Below is main process in which above four types of features extracted form hybrid feature codebook of size 91 .

\section{Algorithm 1: Main Feature extraction algorithm}

Input: Segmented Handwritten Image

Step 1: Extract Statistical/Geometric Features from Input and form feature vector $\mathrm{GeF}$

Step 2: Extract Regional/Structural Features from Input and form feature vector ReF

Step 3: Extract Gradient Features: Gradient and Direction

Step 3.1: Apply mean and standard deviation on gradient

Step 3.2: Apply mean and standard deviation on direction

Step 3.3: Form final 4 features gradient vector called $\mathrm{GrF}$

Step 4: Extract Distance Transform from Input.

Step 4.1: Apply mean and standard deviation on distance transform

Step 4.2: Form final 2 features distance transform vector called DiF.

Step 5: Combine GeF, ReF, GrF and $\mathrm{DiF}$ to form 91 feature vectors called $\mathrm{CHF}$.

Output: Feature Vector CHF.

\subsection{Classification: Methods Used}

Below figure 2 is showing complete flowchart of online handwritten recognition system. Here FFNN classifier is proposed to be used as compared to existing KNN and SVM. In this section all the related classifiers are discussed in details.

\subsubsection{Support Vector Machine:}

Author Vapnik initially presented the support vector machine [9] for classification and recognition problem solving. SVM has many important features supported with good empirical results. SVM is supporting well for classification and estimation of non-linear function. Time series prediction is done by SVM and hence comparable to another radial basis function. The important parameters to decide the SVM performance are selection of kernel function as well as penalty parameter. For the proposed work SVM is out-ofscope of this paper as proposed work is focused on FFNN. For comparative study SVM is used with radial basis kernel function for recognition of handwritten image characters. The mapping of nonlinear samples to higher dimensional space is done by RBF kernel. Below listed are merits and demerits of using SVM.

Merits

- Efficiently works for spaces with high dimension.

- Efficiently works for conditions in which dimension numbers are more than the total samples.

- Training point's subset is used during the decision function which reduces the memory requirement.

- $\quad$ SVM is versatile and flexible to use.

Demerits

- SVM performs poor in condition in which total features is more than the total samples.

- Probability estimations are not directly given by SVM; rather it is calculated using complex method.

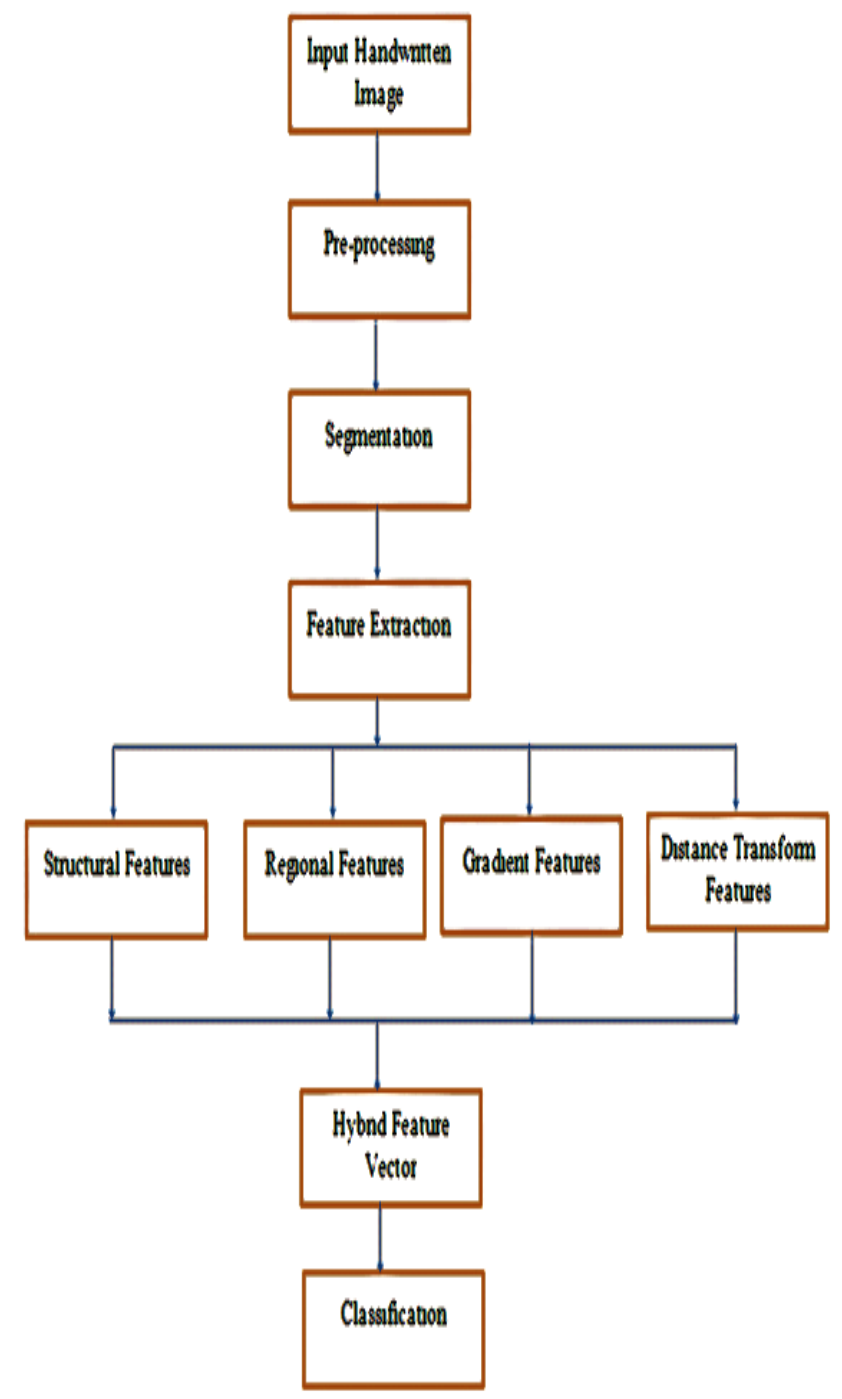

Figure 1: Proposed System Architecture

\subsubsection{K-Nearest Neighbor [KNN]}

This is another classification and recognition method in pattern recognition domain. $\mathrm{KNN}$ is nothing but the nonparametric technique introduced for regression and 
classification. For both regression and classification, KNN takes input consisting of $\mathrm{K}$-nearest samples of training features. KNN result is depending on its use either for classification or regression

KNN Classification: In this input test sample is classified to class membership based on training samples features matching using Euclidian distance or any distance method. The closest match of test sample with training sample is considered as its detected, recognized and classified class of membership.

KNN Regression: the result is belongs to the object property value. This value is computed from the mean of values of its nearest neighbors.

Merits: The advantage of using KNN classifier is that it is very easy and working good for simple problems of recognition.

Demerits: KNN classifier is lazy learner which is main demerit. Failed to learn anything from the training features space which results that $\mathrm{KNN}$ is not robust for noisy data and not generalized.

\section{FEED FORWARD NEURAL NETWORK (FFNN)}

The FFNN is nothing but biologically motivated approach of classification. FFNN is composed of large number of simple processing units those are organized in layers. Each unit in current layer is connected with all other previous layer units. Every connection may have varying weight or strength; hence there is no possibility of similarity between all connections. Network knowledge is encoded into the weights on such connections. Commonly neural network units are known as nodes. In FFNN, data feeding is done at inputs and then passing through network. This data passing is done layer by layer, until data received at outputs. When FFNN acts as classifier, there is no feedback mechanism among layers. Therefore such classifier is knows as feed forward neural network classifier. Below figure 2 is showing example of FFMM in which number of layers are 2, number of units at outputs is 5 , number units at hidden layer is 4 , and number of units at input is 3 . Below listed are advantages of using FFNN for online handwritten character recognition:

- FFNN applicable for different problems.

- Can be applied to problems, for which analytical methods do not yet exist

- $\quad$ Can be used to model non-linear dependencies.

- If there is a pattern, then neural networks should quickly work it out, even if the data is 'noisy'.

- Always gives some answer even when the input information is not complete.

- Networks are easy to maintain.

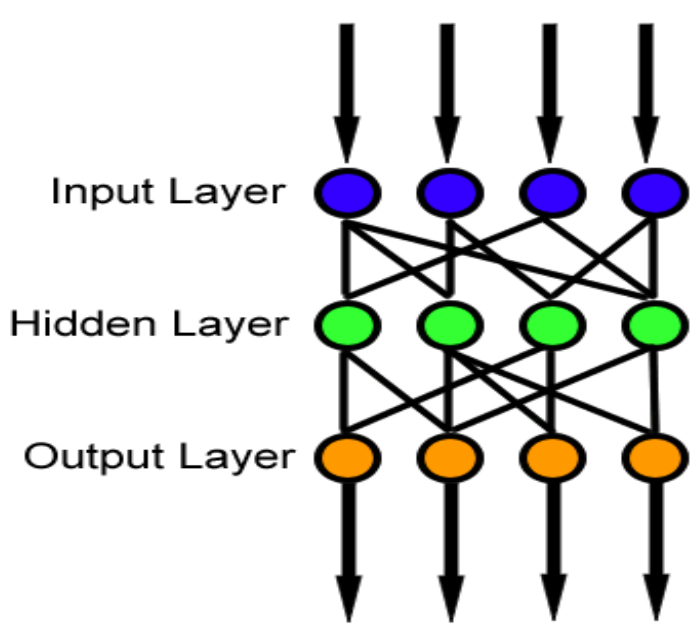

Figure 2: Example of FFNN

For proposed work FFNN is used with below settings and inputs.

1. Learning method $=$ Competitive Learning

2. Number of Hidden Layer $=5,10,15,20,25,30$

3. Type of Neural Network $=$ Feed-forward

4. Input Neurons [Number of input layers] $=91$

5. Output Neurons [Number of output layers] $=3$

6. Learning Constant Value $[\mathrm{C}]=1$

Below are algorithms for both training and recognition using FFNN.

\section{Algorithm 2: Training Algorithm}

Step 1: Input feature matrix reading at layer $1 \mathrm{Fi}$.

Step 2: Computation of activation value for every neuron [ANi].

Step 3: Searching neuron with maximum ANi value.

Step 4: Extract the step 3 results with its input_id and max_ANi_index.

Step 5: Output Ok is set to 1 for kth neuron who's having maximum ANi value.

Step 6: Else set output to 0 .

Step 7: Feed the input of previous layer to next layer still to the output layer.

Step 8: Repeat above steps for all input layers.

\section{Algorithm 3: Recognition Algorithm}

Step 1: Read test pattern to be recognized or classified

Step 2: Compute activation value ANi during layer 2

Step 3: Selecting neuron with max ANi.

Step 4: Neuron with max ANi index is extracted and save it as input_id and max_ANi_index for purpose of matching.

Step5: If match is successful, then input_id of max ANi is returned as output.

Step6: Stop 


\section{PRACTICAL RESULTS AND ANALYSIS}

The current results and performance on input 23 handwritten images of three different candidates using FFNN, KNN and SVM. The output of phase 1 and 2 is showing in below figure 3:

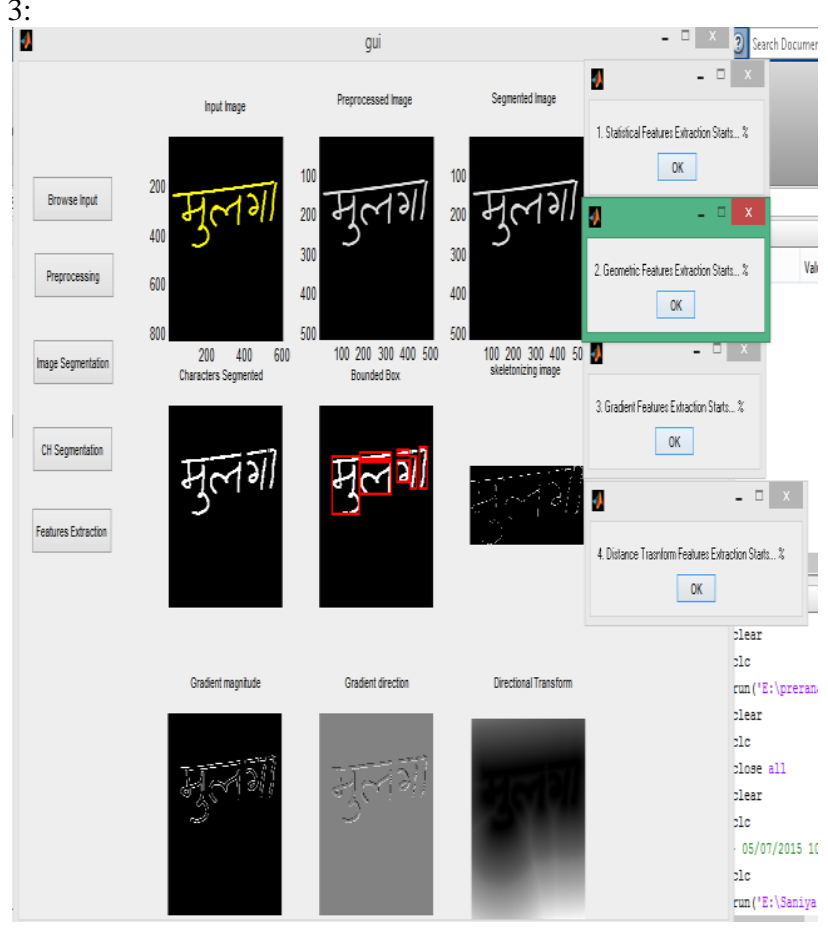

Figure 3: Example Devanagari script

Further for comparative analysis performance evaluation based on recognition accuracy and recognition time is presented.

\subsection{Varying Hidden Layers}

As currently dataset is limited the effect of changing the number input hidden layers for classification and recognition is checked. Below table 1 is showing the results for correction and incorrect classification according to different number of input hidden layers.

Table 1: Performance of FFNN against number of hidden layers

\begin{tabular}{|c|c|c|c|}
\hline $\begin{array}{c}\text { Number } \\
\text { of hidden } \\
\text { layers }\end{array}$ & $\begin{array}{c}\text { Correct } \\
\text { Classification } \\
{[\%]}\end{array}$ & $\begin{array}{c}\text { Incorrect } \\
\text { Classification } \\
{[\%]}\end{array}$ & $\begin{array}{c}\text { Time } \\
{[\text { Seconds] }}\end{array}$ \\
\hline 5 & 91.304348 & 8.695652 & 0.833922 \\
\hline 10 & 91.304348 & 8.695652 & 1.959070 \\
\hline 15 & 78.260870 & 21.739130 & 3.189879 \\
\hline 20 & 78.260870 & 21.739130 & 4.947593 \\
\hline 25 & 78.260870 & 21.739130 & 20.663303 \\
\hline 30 & 82.608696 & 17.391304 & 12.248829 \\
\hline
\end{tabular}

From above table it is showing that performance of FFNN is getting better when number of input layers either 5 or 10 with less time for training and recognition processes. As the number of neurons are increasing, performance of accuracy is decreasing with required time is increasing.

\subsection{Comparing Performance}

Below table 2 is showing the comparative study for accuracy is using FFNN, KNN and SVM classifiers on 100-images training dataset. From table 2 below, it is showing that performance of FFNN giving better accuracy of classification with less time of recognition and training.

Table 2: Performance of FFNN against SVM and KNN

\begin{tabular}{|c|c|c|c|}
\hline $\begin{array}{c}\text { Classificatio } \\
\mathbf{n} \text { Method }\end{array}$ & $\begin{array}{c}\text { Correct } \\
\text { Classificatio } \\
\mathbf{n} \\
{[\boldsymbol{\%}]}\end{array}$ & $\begin{array}{c}\text { Incorrect } \\
\text { Classificatio } \\
\mathbf{n} \\
{[\boldsymbol{\%}]}\end{array}$ & $\begin{array}{c}\text { Time } \\
\text { [Seconds } \\
]\end{array}$ \\
\hline SVM & 86.34 & 13.66 & 1.03 \\
\hline KNN & 79.1 & 20.9 & 2.1 \\
\hline FFNN & 91.3 & 8.7 & 0.83 \\
\hline
\end{tabular}

6. CONCLUSION AND FUTURE WORK

On-going research on online Devanagari handwritten character recognition is further introduced in this paper with different classifiers and claims their accuracy through extensive practical results. This paper proposed final framework and methodology for handwritten recognition for Devanagari script using combination of different features, appropriate segmentation method, and efficient FFNN classifier. In this paper the performance of system using limited dataset of 100 images is evaluated. For future work, large number of image datasets needs to be evaluated and tested with some more performance metrics.

\section{REFERENCES}

[1] Prof. S.P.Kosbatwar, Prof.S.K.Pathan,: "Pattern Association for character recognition by BackPropagation algorithm using Neural Network approach" International Journal of Computer Science \& Engineering Survey (IJCSES) Vol.3, No.1, February 2012.

[2] Kauleshwar Prasad, Devvrat C. Nigam, Ashmika Lakhotiya: Character Recognition Using Matlab"s Neural Network Toolbox, International Journal of u- and e- Service, Science and Technology Vol. 6, No. 1, February, 2013.

[3] Sunit Bandaru "Handwritten character recognition using neural network"

[4] Muhammad Naeem Ayyaz, Imran Javed and Waqar Mahmood 1 2: Handwritten Character Recognition Using Multiclass SVM Classification with Hybrid Feature Extraction, Pak. J. Engg. \& Appl. Sci. Vol. 10, Jan., 2012 (p. 57-67).

[5] Anita Pal \&Dayashankar Singh, "Handwritten English Character Recognition Using Neural Network"International Journal of Computer Science \& Communication Vol. 1, No. 2, July-December 2010, pp. 141-144.

[6] U. Bhattacharya, B. B. Chaudhuri, R. Ghosh and M. Ghosh, "On Recognition of Handwritten Devnagari Numerals", In Proc. of the Workshop on Learning Algorithms for Pattern Recognition (in conjunction with 
the 18th Australian Joint Conference on Artificial Intelligence), Sydney, pp.1-7, 2005.

[7] N. Sharma, U. Pal, F. Kimura, and S. Pal," Recognition of Off-Line Handwritten Devnagari Characters Using Quadratic Classifier", ICVGIP 2006, LNCS 4338, pp. $805-816,2006$.

[8] Vedgupt Saraf, D.S. Rao, "Devnagari Script Character Recognition Using Genetic Algorithm for Get Better Efficiency", International Journal of Soft Computing and Engineering (IJSCE) ISSN: 2231-2307, Volume-2, Issue-4, April 2013.
[9] V.N. Vapnik, The Nature of Statistical Learning Theory, 2nd ed., Springer, 2000.

[10] Y. Yang, Expert Network: Effective and Efficient Learning from Human Decisions in Text Categorization and Retrieval. Proc. 17th Annual Intl. ACM SIGIR Conf. Research \& Development in Information Retrieval, Dublin (Ireland), pp. 13 - 22, 1994.

[11] Y. Yang, and C.G. Chute, An Example-based Mapping Method for Text Classification and Retrieval. ACM Trans. Information Systems, vol. 12, no. 3, pp. $252-$ 277, 1994. 